\title{
Data transmission capabilities of silicon avalanche mode light-emitting diodes
}

\author{
Vishal Agarwal, Anne-Johan Annema, Raymond J. E. Hueting, Satadal Dutta, Lis K. Nanver, and Bram Nauta
}

\begin{abstract}
The data transmission capabilities of silicon avalanche mode light-emitting diodes (AMLEDs) were investigated and the results are correlated to the multiplication noise and leakage current. The incoming data were modulated using pulse position modulation and the bit-error-rate (BER) and jitter in the transmitted data were measured. The results indicate an intrinsically low speed in terms of BER and jitter. From various size AMLEDs, temperature variations and optical excitations, it is shown that the speed can be improved by using AMLEDs with a (1) relatively high multiplication noise, (2) relatively high leakage current and (3) higher charge-per-bit. Design recommendations for the high speed AMLEDs are discussed.
\end{abstract}

Index terms - Avalanche light emitting diodes, Data communication, Modulation speed, Pulse position modulation.

\section{INTRODUCTION}

The significant overlap of the emission spectrum of silicon (Si) avalanche mode light-emitting diodes (AMLEDs) with the responsivity of Si photodetectors (PDs) makes them attractive for monolithic integration of optocouplers in standard CMOS [1]-[4]. In [5], we have demonstrated optocoupling between Si AMLEDs and PDs in standard CMOS and analyzed the effects of the link length on the optocoupling efficiency. For digital data communication, the output of an AMLED needs to be modulated in response to the incoming data. The maximum data rate in such a system is typically limited by how fast the LED can be turned on and off [6], [7].

The small signal modulation speed of AMLEDs has been reported in tens of $\mathrm{GHz}$ [8], [9], which is determined by the transit time $\tau_{\mathrm{t}} \sim w_{\mathrm{d}} / v_{\mathrm{sat}}$, where $w_{\mathrm{d}}$ is the depletion region width and $v_{\text {sat }} \sim 10^{7} \mathrm{~cm} / \mathrm{s}$ is the saturation velocity of electrons and holes; $\tau_{t}$ is typically a few tens of ps. However, the large signal switching speed depends on the availability of free carriers in the multiplication region and triggering of a new avalanche event. Without any experimental proof, it was claimed that this speed is also in tens of $\mathrm{GHz}$ [10]. We show that triggering of a new avalanche event can be a relatively slow process. If an avalanche would not be triggered during transfer of a data bit, it leads to a bit-error in the communication link. In addition, the delay variation in turning on of avalanche leads to jitter in the transmitted data. Obviously,

Manuscript changed August 28, 2018, v1.0.

This work is funded by the Applied and Engineering Sciences (TTW) division of the Netherlands Organization for Scientific Research (NWO).

V. Agarwal (e-mail: v.agarwal@utwente.nl), A.J. Annema and B. Nauta are with the IC Design group, University of Twente, Enschede, The Netherlands. R.J.E. Hueting and L. K. Nanver are with the MESA+ Institute for Nanotechnology, University of Twente, Enschede, The Netherlands.

S. Dutta is with Lionix International B.V., The Netherlands.

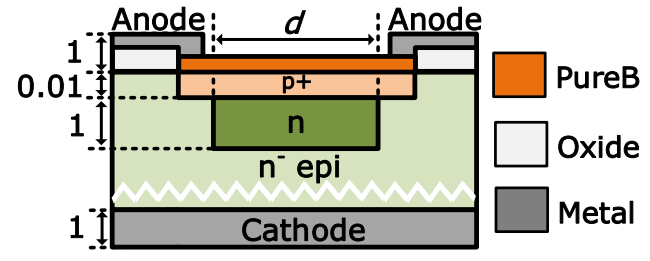

Fig. 1. Schematic cross-section of a PureB AMLED (not to scale, all dimensions in $\mu \mathrm{m}$ ). The oxide is $\sim 0.3 \mu \mathrm{m}$ thick. Diameter $(d)$ of an AMLED is defined by the dimensions of the $\mathrm{n}$-enhancement layer.

a high bit-error-rate (BER) and jitter are undesirable. In this work, it is shown that:

- AMLEDs with more defects have more multiplication noise and achieve higher speed.

- AMLEDs with a relatively high leakage current have a high number of free carriers to trigger an avalanche, which improves speed.

- Higher speed can also be obtained by driving AMLEDs at a higher bias, which increases the avalanche triggering probability, at the cost of increased power consumption.

Based on these results, design recommendations for high speed AMLEDs are discussed. The presented results are important for the implementation of low power optical links using AMLEDs [3]-[5], [11].

\section{EXPERIMENTAL AMLEDS AND NOISE}

For our experiments, the AMLEDs were fabricated in a pure boron (PureB) technology, a schematic cross-section of which is shown in Fig. 1. The anode was formed by chemical vapor deposition of PureB in windows to the $\mathrm{n}-\mathrm{Si}$ surface through an oxide layer. The $\mathrm{n}-\mathrm{Si}$ substrate had a nominal doping concentration of $10^{15} \mathrm{~cm}^{-3}$ and an n-enhancement layer was formed under the anode by implanting phosphorus to achieve a local doping concentration of $10^{17} \mathrm{~cm}^{-3}$. A detailed description of the processing is given in [12] where similar PureB diodes were fabricated with the addition of a buried $\mathrm{n}^{+}$ layer. Four circular diodes, on the same die were selected with diameters $(d)$ of $8 \mu \mathrm{m}, 15 \mu \mathrm{m}, 20 \mu \mathrm{m}$ and $30 \mu \mathrm{m}$; we label these diodes as $\mathrm{J} 8, \mathrm{~J} 15, \mathrm{~J} 20$ and $\mathrm{J} 30$ respectively.

Fig. 2 shows the DC $I-V$ characteristics of the AMLEDs measured using a Keysight B2901A Source/Measure Unit (SMU) and 1 second integration time. The breakdown voltage $\left(V_{\mathrm{BR}}\right)$ of the AMLEDs, defined as the reverse voltage $\left(V_{\mathrm{R}}\right)$ at which the reverse current $\left(I_{R}\right)$ starts to sharply increase, is $\sim 13.7 \mathrm{~V}$. An example of the light-emission from such an AMLED is also shown in the inset.

We have shown that in avalanche diodes, $I_{\mathrm{R}}$ exhibits Random Telegraph Signal (RTS) phenomena in the steep part of 


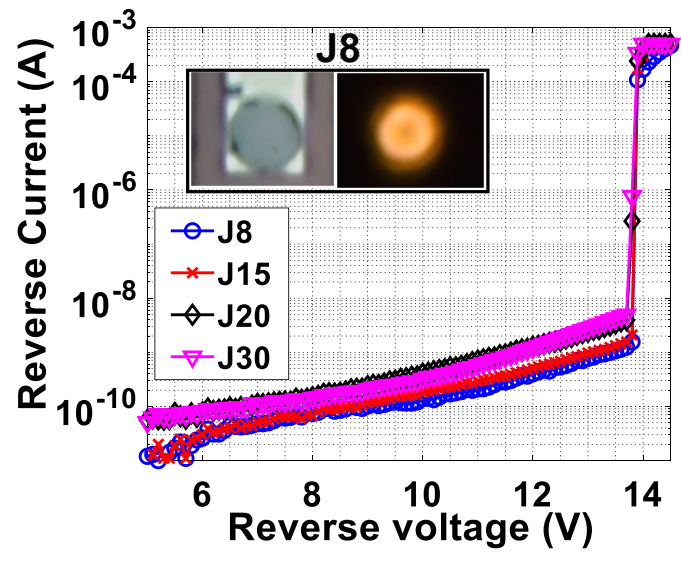

Fig. 2. Measured reverse $I-V$ characteristics of PureB AMLEDs. Inset shows light emission from $\mathrm{J} 8$, biased at a reverse current of $1 \mathrm{~mA}$, captured using a Nikon D3100 camera with an integration time of 30 seconds.
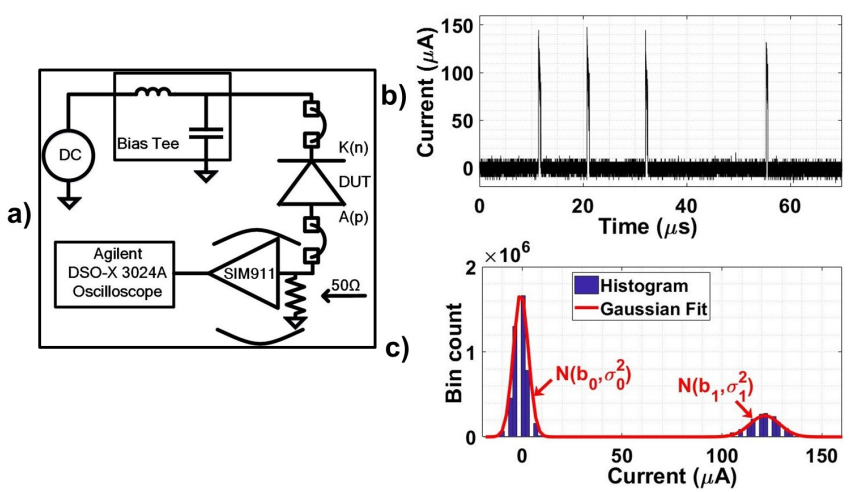

Fig. 3. (a) Random Telegraph Signal (RTS) measurement setup. (b) Measured RTSs in $I_{R}$. (c) Histogram of the measured RTSs. $\sigma_{0}$ is the noise from the experimental setup whereas $\sigma_{1}$ also includes multiplication noise $\left(\sigma_{\mathrm{M}}\right)$.

the $I-V$ characteristics [13]. Initially close to $V_{\mathrm{BR}}$, the RTSs are short in duration. As the $V_{\mathrm{R}}$ increases, the RTSs become longer and their amplitude increases [14]. Ultimately, the RTSs disappear and the diode is in a continuously on-state. In the on-state, there is also multiplication noise $\left(\sigma_{\mathrm{M}}\right)$ present in the avalanche current [14], [15].

The RTS phenomena were measured as done previously (Fig. 3) [14]. We define $\sigma_{M}$ as the difference between the noise in the on-state of the device $\left(\sigma_{1}\right)$ and the measurement setup noise $\left(\sigma_{0}\right): \sigma_{\mathrm{M}}=\sqrt{\sigma_{1}^{2}-\sigma_{0}^{2}}$ (Fig. 3c) [15].

Fig. 4 shows an example of the measured transient $I_{\mathrm{R}}$ for all AMLEDs in a continuous on-state. Fig. 5 shows the estimated $\sigma_{\mathrm{M}}$ for all AMLEDs as a function of their diameter $(d)$. A higher $\sigma_{\mathrm{M}}$ can be observed for larger AMLEDs which can be explained by the higher amount of defects in a larger device [14]; the noise generated by each defect is added up [16]. The linear dependency of $\sigma_{\mathrm{M}}$ on $d$ indicates that the number of defects increases linearly with the area of the diode.

\section{Pulse POSition MOdUlation SPEED}

\section{A. AMLED driver circuit}

Light is emitted from an AMLED only during avalanche [17]. For low power receivers in digital optical links using e.g. single-photon avalanche diodes, the LED should be switched between completely on and off conditions. Therefore, the
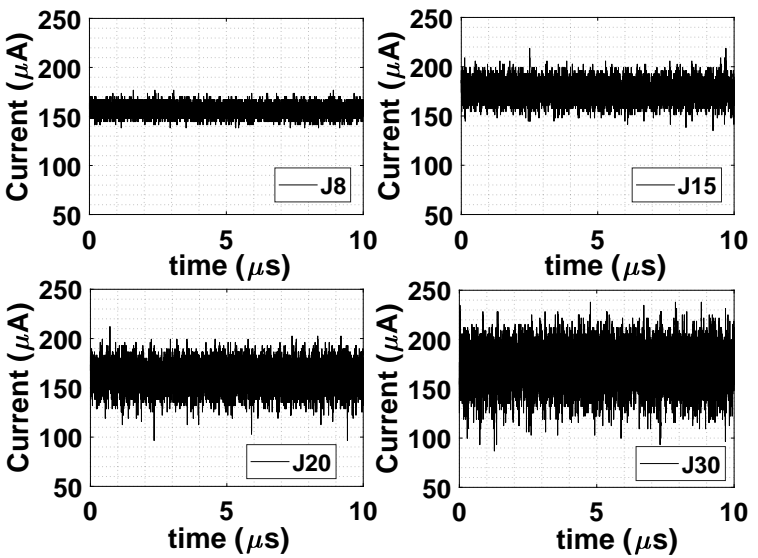

Fig. 4. Example of measured transient avalanche currents when the AMLEDs are in on-state; these data include the measurement setup noise $\left(\sigma_{0}\right)$.

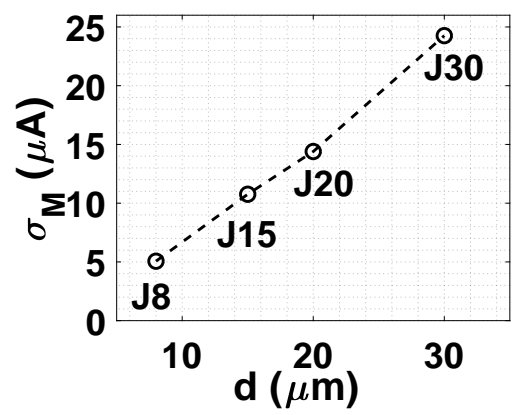

Fig. 5. Multiplication noise $\left(\sigma_{M}\right)$ as a function of diameter $(d)$ of AMLEDs. voltage across an AMLED ( $\left.V_{\text {AMLED }}\right)$ should be modulated between below and above $V_{\mathrm{BR}}$ by driver circuit.

Fig. 6 shows the schematics of the driver circuit along with illustrative transient waveforms that we used to modulate $V_{\text {AMLED }}$. The circuit can also limit the charge-per-bit $\left(Q_{\mathrm{b}}\right)$ through the AMLED. Limiting $Q_{\mathrm{b}}$ limits the energy-per-bit $\left(E_{\mathrm{b}}\right)$, which is an important figure-of-merit in optical links.

We briefly describe the functionality of this circuit. For further details of this circuit, we refer the reader to [18], in which we have demonstrated an integrated implementation of this driver circuit. Initially the AMLED is biased below $V_{\mathrm{BR}}$ and then $V_{\mathrm{AMLED}}=V_{\mathrm{LOW}}=V_{\mathrm{BIAS}}-V_{\mathrm{DD}}$. First the reset switch M1 is opened using the control signal RST. Then the control signal IN is set high and $V_{\mathrm{AMLED}}$ initially increases to:

$$
V_{\mathrm{AMLED}}=V_{\mathrm{BIAS}}-V_{\mathrm{DD}} \cdot\left(\frac{C_{\mathrm{P}}}{C_{\mathrm{P}}+C_{\mathrm{Q}}}\right)
$$

where the total parasitic capacitance $C_{\mathrm{P}}=C_{\mathrm{PAR}}+C_{\mathrm{AMLED}}$, with $C_{\mathrm{PAR}}$ the circuit parasitic capacitance and $C_{\mathrm{AMLED}}$ the AMLED capacitance. The initial excess bias $\left(V_{\mathrm{EX}, \mathrm{i}}=\right.$ $\left.V_{\mathrm{AMLED}}-V_{\mathrm{BR}}\right)$ is:

$$
V_{\mathrm{EX}, \mathrm{i}}=V_{\mathrm{BIAS}}-V_{\mathrm{DD}} \cdot\left(\frac{C_{\mathrm{P}}}{C_{\mathrm{P}}+C_{\mathrm{Q}}}\right)-V_{\mathrm{BR}} \text {. }
$$

The initial $I_{\mathrm{AMLED}} \approx V_{\mathrm{EX}, \mathrm{i}} / R_{\mathrm{AMLED}}$ where $R_{\mathrm{AMLED}}$ is the resistance of the AMLED, and $I_{\mathrm{AMLED}}$ charges $C_{\mathrm{Q}}$, thereby reducing $V_{\mathrm{AMLED}}$ and $I_{\mathrm{AMLED}}$. As $V_{\mathrm{AMLED}}$ reduces to $V_{\mathrm{BR}}$, the avalanche is quenched. The maximum $Q_{\mathrm{b}}$ equals $C_{\mathrm{Q}} V_{\mathrm{EX}, \mathrm{i}}$ :

$$
Q_{\mathrm{b}}=\int_{0}^{T_{\mathrm{ON}}} I_{\mathrm{AMLED}}(t) d t \leq C_{\mathrm{Q}} V_{\mathrm{EX}, \mathrm{i}} \leq C_{\mathrm{Q}} V_{\mathrm{DD}}
$$




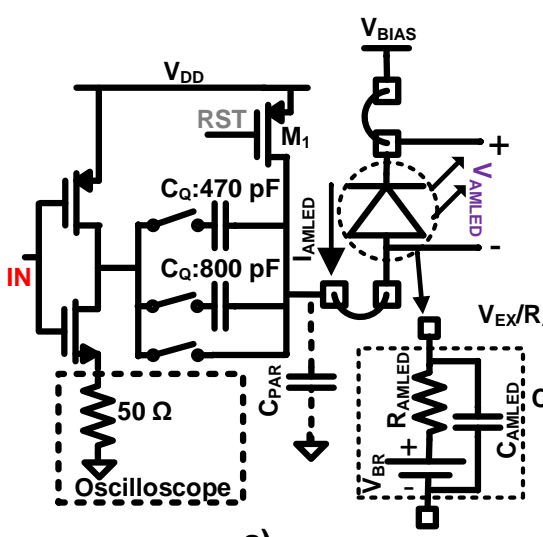

a)

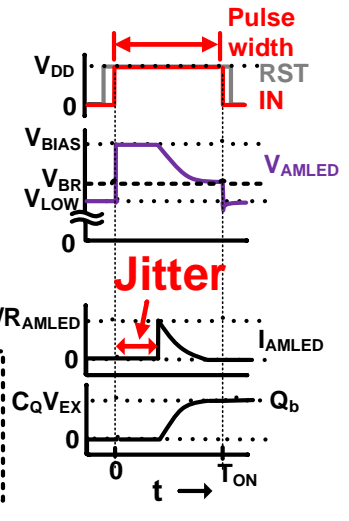

b)
Fig. 6. (a) Schematic layout of an AMLED driver circuit to measure modulation speed [18]. The $I_{\mathrm{AMLED}}$ was measured directly on the oscilloscope. In our experimental setup, three $C_{\mathrm{Q}}$ settings were incorporated: $470 \mathrm{pF}, 800$ $\mathrm{pF}$ and short $\left(C_{\mathrm{Q}} \rightarrow \infty\right)$. A simplified model for the AMLED is also shown [18]. (b) Illustrative transient waveforms.

where $T_{\mathrm{ON}}$ is the pulse width of the control signal IN.

Due to the relatively low internal quantum efficiency of AMLEDs $\left(10^{-5}\right)$ [18], high speed optical measurements are not possible using conventional photodetectors. Here, we measure $I_{\mathrm{AMLED}}$ as an indication of the light emission. The relation between $I_{\mathrm{AMLED}}$ and the light emission from AMLEDs is discussed in section III-G.

In this work, the circuit of Fig. 6(a) was designed on a printed circuit board (PCB) which, in contrast to the CMOS integrated implementation of [18], resulted in $C_{\mathrm{P}} \sim 20 \mathrm{pF}$.

\section{B. Pulse position modulation and bit error rate}

AMLEDs have a relatively low efficiency and therefore for fast and low power receivers, they should be integrated with highly sensitive photodetectors such as single-photon avalanche diodes (SPADs) [19]. SPADs are p-n junctions biased above breakdown where an incoming photon can trigger an avalanche. The current then swiftly rises to macroscopic levels which can be detected using readout circuits [20].

For data communication applications using SPADs, pulse position modulation (PPM) is a modulation scheme of choice [19]. Fig. 7 illustrates the transient waveforms of the control signal IN of our driver circuit for a two level PPM. In the transmitter, if an avalanche is not triggered during a data bit, there is no light emission and consequently a bit error occurs in the transmitted data. Therefore, the BER is defined as the fraction of the number of data bits where no avalanche was detected, as illustrated in Fig. 7 (Note that BER $>0.5$ is possible according to this definition).

We varied $V_{\mathrm{LOW}}$ by adjusting $V_{\mathrm{BIAS}}$ and $V_{\mathrm{DD}}$ in the driver circuit. As a result, the leakage current of the AMLED in the off-state is tuned. $V_{\mathrm{BIAS}}$ and $V_{\mathrm{DD}}$ were also adjusted to achieve different $V_{\mathrm{EX}, \mathrm{i}}$ for each $V_{\mathrm{LOW}}$ (Eq. (2)). We will show in section III-D that as $V_{\text {LOW }}$ or $V_{\mathrm{EX}, \mathrm{i}}$ increases, the BER decreases.

Furthermore, to study the effect of varying $Q_{\mathrm{b}}$, the $C_{\mathrm{Q}}$ was varied between three settings (Fig. 6(a)): $470 \mathrm{pF}, 800 \mathrm{pF}$ and $C_{\mathrm{Q}} \rightarrow \infty$ (implemented as a short yielding no quenching). During an avalanche event, some of the carriers flowing through
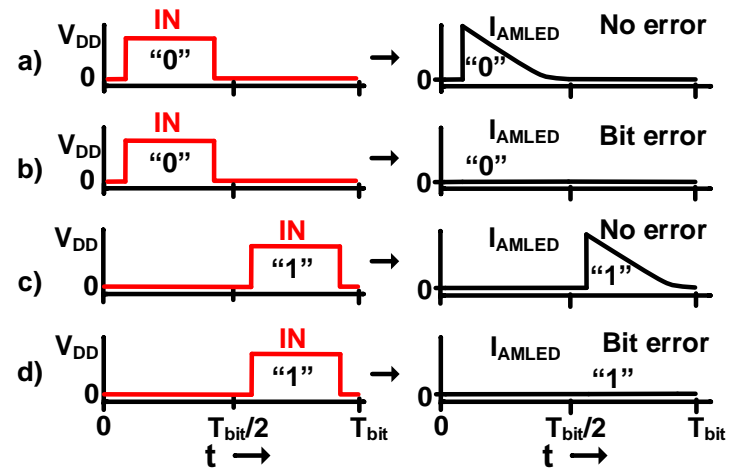

Fig. 7. (Left) Illustrative transient waveforms of the control signal IN for bit " 0 " in (a,b) and for bit " 1 " in (c,d) when data are modulated using a two-level PPM; $T_{\text {bit }}=1 / f_{\mathrm{s}}$ is the bit duration time. (Right) Illustrative example of BER: For bit "0", avalanche is triggered in (a) and therefore there is no bit error in the transmitted data whereas avalanche is not triggered in (b) causing a bit error. Similarly for bit " 1 ", there is no bit error in (c) whereas (d) causes a bit error. Hence, (b) and (d) contribute to BER.

\begin{tabular}{|c|c|c|c|}
\hline $\mathbf{V}_{\mathbf{D D}}(\mathbf{V})$ & $\mathbf{V}_{\mathbf{B I A S}}(\mathbf{V})$ & $\mathbf{V}_{\text {LOW }}(\mathbf{V})$ & $\sim \mathbf{V}_{\mathbf{E X}, \mathbf{i}}(\mathbf{V})$ \\
\hline 6 & 17.6 & 11.6 & 3.9 \\
\hline 7 & 18.6 & 11.6 & 4.9 \\
\hline 8 & 19.6 & 11.6 & 5.9 \\
\hline 5 & 17.6 & 12.6 & 3.9 \\
\hline 6 & 18.6 & 12.6 & 4.9 \\
\hline 7 & 19.6 & 12.6 & 5.9 \\
\hline 4 & 17.6 & 13.6 & 3.9 \\
\hline 5 & 18.6 & 13.6 & 4.9 \\
\hline 6 & 19.6 & 13.6 & 5.9 \\
\hline
\end{tabular}

Fig. 8. Bias settings of the driver circuit. For all three $C_{\mathrm{Q}}$ settings, by tuning $V_{\mathrm{DD}}$ and $V_{\mathrm{BIAS}}$, different values of $V_{\mathrm{LOW}}$ and $V_{\mathrm{EX}, \mathrm{i}}$ are obtained.

the depletion region of the AMLED can get trapped. These trapped carriers are randomly released, causing free carriers that may trigger another avalanche event. This phenomenon is well-known as "afterpulsing" in SPADs [20], and is an undesired phenomenon in SPADs limiting their count rate [20]. However as shown in section III-D, the mechanism yielding afterpulsing in SPADs helps to reduce the BER in AMLEDs.

To test the performance of the AMLEDs, a Pseudo Random Bit Sequence (PRBS) with a length of $2^{10}$ bits was generated. The control signals (IN and RST in Fig. 6(b)) for PRBS data were generated using a Keysight 33200A dual channel arbitrary waveform generator at a data rate $\left(f_{\mathrm{s}}\right)$ of $100 \mathrm{kbps}$.

Fig. 8 summarizes all biasing settings used in our measurements. The transient data at the oscilloscope were acquired at $2 \mathrm{GS} / \mathrm{s}$ ( $0.5 \mathrm{~ns}$ resolution). The performance of the AMLEDs was measured in terms of jitter and BER. The jitter is defined as the time delay between the time at which $V_{\mathrm{AMLED}}>V_{\mathrm{BR}}$ and the turn-on of avalanche, see Fig. 6(b). In our measurements, due to the used PRBS, the BER can be reliably measured down to $10^{-3}$ and therefore is lower limited to $10^{-3}$.

\section{Functionality}

Fig. 9 demonstrates an example of the measured $I_{\mathrm{AMLED}}$ for J8 and J30; J8 shows a larger BER $(\sim 0.98)$ than J30 


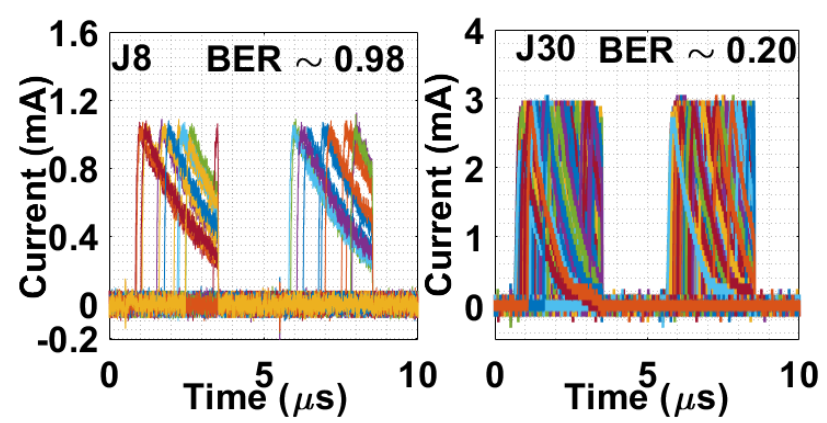

Fig. 9. Measured transient waveforms of $I_{\mathrm{AMLED}}$ at $C_{\mathrm{Q}}=800 \mathrm{pF}, V_{\mathrm{BIAS}}=$ $17.6 \mathrm{~V}$ and $V_{\mathrm{DD}}=5 \mathrm{~V}$ for $\mathrm{J} 8$ and $\mathrm{J} 30$. The data have been superimposed for all $2^{10}$ bits. Y-axis scales are different for clarity.

$(\sim 0.2)$ because of a higher multiplication noise in the latter (see section III-D).

An ideal probability density function (PDF) of jitter for a two level PPM would be two dirac delta functions at the time instants of the rising edge of $V_{\text {AMLED }}$ (Fig. 6(b) and Fig. 7). However, in Fig. 9 large jitter in the turn-on time in both AMLEDs can be observed. This is because of a lack of free carriers and a defect count in these devices to trigger an avalanche. The PDFs of jitter are used in sections III-E, III-F and IV-A to demonstrate the effect of the leakage current and defects on the speed of AMLEDs.

\section{D. $B E R$}

The probability of triggering of an avalanche during a pulse when $V_{\mathrm{AMLED}}>V_{\mathrm{BR}}$ depends on the number of free carriers in the depletion region $\left(N_{\mathrm{d}}\right)$ and the probability that a free carrier triggers avalanche $\left(P_{\mathrm{a}}\right)$. For a related analysis, a model of the triggering phenomenon was reported to explain the dark count rate of the SPADs [21]. In that model, the arrival of free carriers in the depletion region was modeled by a Poisson process. The probability that an avalanche is triggered $\left(P_{\mathrm{d}}\right)$ in a pulse of duration $T_{\mathrm{ON}}$ is given by [21]:

$$
\begin{gathered}
P_{\mathrm{d}}=1-\mathrm{e}^{-N_{\mathrm{d}} \cdot P_{\mathrm{a}}}, \\
\text { with } N_{\mathrm{d}}=N_{\mathrm{d} 1}+N_{\mathrm{d} 2}+N_{\mathrm{t} 1}+N_{\mathrm{t} 2}+N_{\mathrm{opt}}, \\
\text { and } \quad P_{\mathrm{a}}=1-\mathrm{e}^{-\frac{V_{\mathrm{EX}}}{\eta V_{\mathrm{BR}}}} . \\
\text { Further, } \quad B E R=1-P_{\mathrm{d}}=\mathrm{e}^{-N_{\mathrm{d}} \cdot P_{\mathrm{a}}} .
\end{gathered}
$$

In the above equations, $N_{\mathrm{d} 1}=I_{\mathrm{R}} T_{\mathrm{ON}} / q_{\mathrm{e}}$ is the number of carriers generated during the pulse where $I_{R}$ is the leakage current and $q_{\mathrm{e}}$ is the elementary charge. Some of the carriers generated before the arrival of the pulse could remain in the depletion region due to the finite bandwidth of the AMLED; $N_{\mathrm{d} 2}$ is the number of those carriers. $N_{\mathrm{t} 1}$ is the number of released carriers from traps during the pulse (causing "afterpulsing" in SPADs). Similar to $N_{\mathrm{d} 2}, N_{\mathrm{t} 2}$ is the number of carriers that are released from traps before the arrival of the pulse. $N_{\mathrm{opt}}$ is the number of optically generated carriers (section III-F). Finally, $\eta$ is a fit parameter that depends on the defect density, temperature and device properties [21].

$N_{\mathrm{d}}$ and $P_{\mathrm{a}}$ are functions of the bias, temperature, device properties and driver circuit operating conditions. Exact solutions of these functions are difficult to obtain. However, the
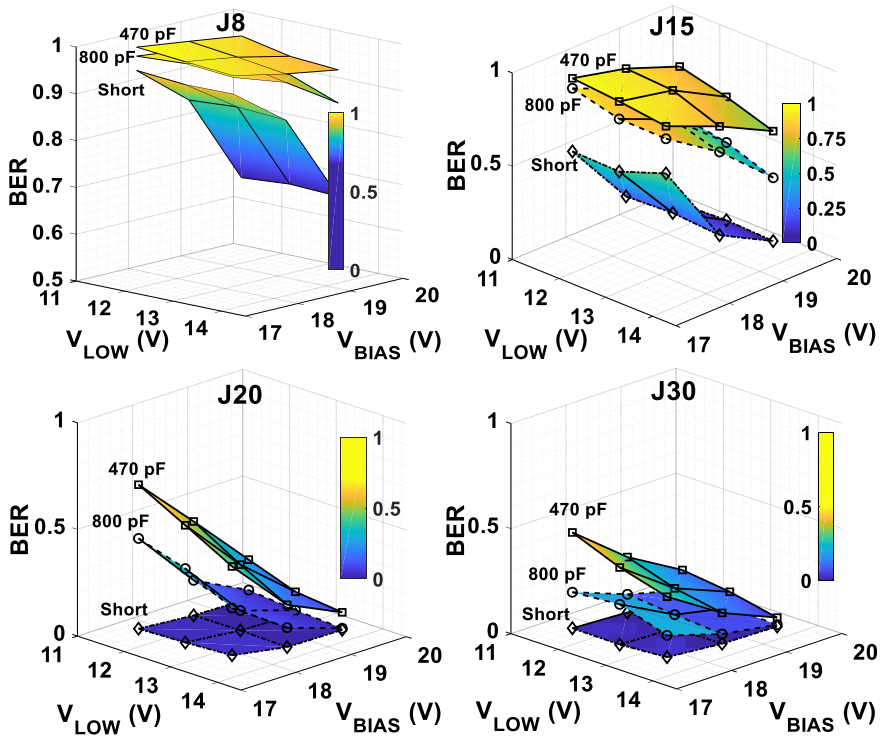

Fig. 10. Measured BER for all AMLEDs as a function of $V_{\text {LOW }}, V_{\text {BIAS }}$ and $C_{\mathrm{Q}}$. BER is shown on a linear scale. For J8, the axis representing BER has a difference scale to enhance clarity.

elegance of this model is its ability to explain the observed trends [21].

Fig. 10 shows the measured BER for all AMLEDs at different bias conditions (Fig. 8) in case of PPM. The BER

1) decreases with increasing $V_{\text {LOw }}$ at a given $V_{\text {BIAS }}$,

2) decreases with increasing $V_{\text {BIAS }}$ at a given $V_{\mathrm{LOW}}$,

3) reduces for a higher $C_{Q}$, and

4) is lower for larger diodes.

The relatively high BER is due to a low defect density, therefore low $P_{\mathrm{a}}$, in these AMLEDs. The defect density is low because of the circular geometry and the presence of the implicit guard ring (Fig. 2) [12].

The decreasing BER for a higher $V_{\text {LOW }}$ (at a fixed $V_{\text {BIAS }}$ ) is because of an increase of $N_{\mathrm{d} 2}$. A higher $V_{\mathrm{BIAS}}$ (at a fixed $V_{\mathrm{LOW}}$ ) causes a higher $N_{\mathrm{d} 1}$ and a higher $P_{\mathrm{a}}$ (Eq. (4c)), consequently resulting in a lower BER.

A higher $C_{\mathrm{Q}}$ causes a higher $Q_{\mathrm{b}}$ (Eq. (3)) and therefore more carriers are trapped during an avalanche event. These trapped carriers can be released during the next bit, increasing the probability of another avalanche. This effect results in a lower BER for a higher $Q_{\mathrm{b}}$.

As the size of an AMLED increases, the multiplication noise increases (Fig. 5) indicating an increasing number of defects for larger diodes. This results in a higher $P_{\mathrm{a}}$ in Eq. 4(d) and therefore in a lower BER for larger devices.

When driving AMLEDs with $C_{\mathrm{Q}} \rightarrow \infty$, the BER further reduces. However, a higher amount of energy is then dissipated in the AMLEDs which increases its temperature. An elevated temperature results in a higher $V_{\mathrm{BR}}$; an example of this selfheating can be observed from the measured $I_{\mathrm{AMLED}}(t)$ in Fig. 11. A higher $V_{\mathrm{BR}}$ reduces $P_{\mathrm{a}}$ (Eq. 4(c)) which tends to increase the BER (Eq. 4(d)). Therefore, BER cannot be reduced by operating AMLEDs at a very high $Q_{\mathrm{b}}$ because of this selfheating effect. 


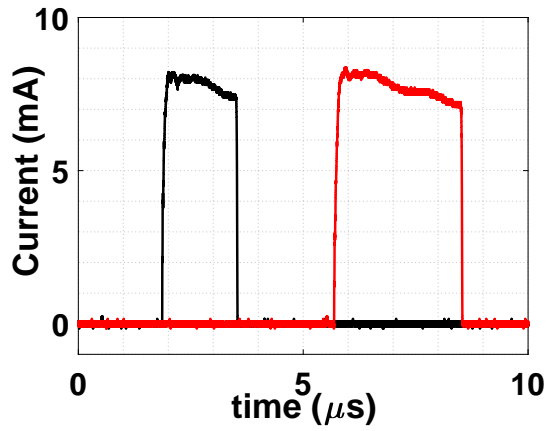

Fig. 11. Example of self heating in J30: $V_{\mathrm{DD}}=8 \mathrm{~V}, V_{\mathrm{BIAS}}=19.6 \mathrm{~V}$ and the driver circuit was operated without any $C_{\mathrm{Q}}$ (short). $I_{\mathrm{AMLED}}$ reduces with time due to the self-heating of the AMLED.

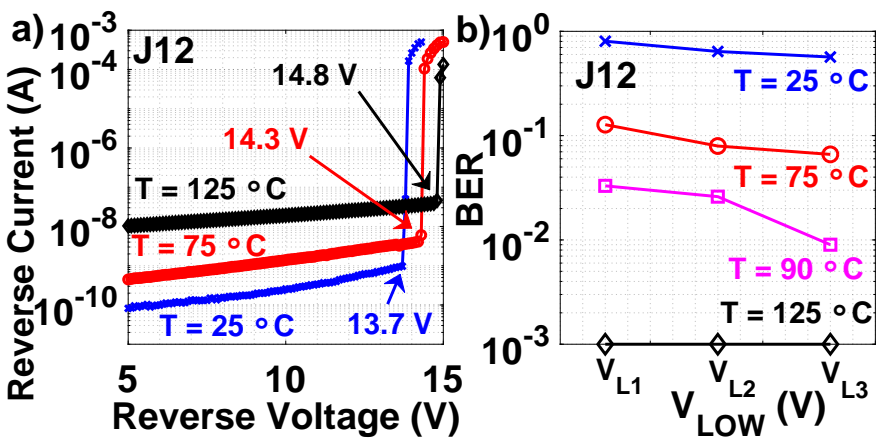

Fig. 12. (a) $I-V$ characteristics of $\mathrm{J} 12$ for three different temperatures. Different $V_{\mathrm{BR}}$ at all temperatures are indicated. (b) Measured BER for diode $\mathrm{J} 12$ at $V_{\mathrm{BIAS}}=V_{\mathrm{BR}}+5.9 \mathrm{~V}, C_{\mathrm{Q}}=470 \mathrm{pF} . V_{\mathrm{L} 1}=V_{\mathrm{BR}}-2.1 \mathrm{~V}, V_{\mathrm{L} 2}=V_{\mathrm{BR}}-1.1$ $\mathrm{V}$ and $V_{\mathrm{L} 3}=V_{\mathrm{BR}}-0.1 \mathrm{~V}$ are the $V_{\mathrm{LOW}}$ at each temperature. The BER is shown on a logarithmic scale for clarity.

In conclusion, for a given AMLED, a reduced $Q_{\mathrm{b}}$ causes a high BER which implies a constraint to reduce $Q_{\mathrm{b}}$ for an optical link ${ }^{1}$. Also, the $Q_{\mathrm{b}}$ cannot be arbitrarily increased because of increasing power consumption and self-heating.

\section{E. Effect of temperature}

The parameters $N_{\mathrm{d}}$ and $P_{\mathrm{a}}$ can be increased by operating the AMLEDs at elevated temperatures. The effect of temperature on the $I-V$ characteristics of a circular $12 \mu \mathrm{m}$ diode (J12) on the same die is shown in Fig. 12(a).

Using the same driver circuit and PRBS data, BER and jitter were measured at three temperatures for J12. By adjusting $V_{\mathrm{BIAS}}$ and $V_{\mathrm{DD}}, V_{\mathrm{LOW}}$ was varied between $V_{\mathrm{BR}}-0.1 \mathrm{~V}, V_{\mathrm{BR}}-$ $1.1 \mathrm{~V}$ and $V_{\mathrm{BR}}-2.1 \mathrm{~V}$ at all temperatures (Fig. 8). Fig. 12(b) shows a lower BER at higher temperatures, which is due to a higher $N_{\mathrm{d}}$ and $P_{\mathrm{a}}$.

Fig. 13 shows an example of the jitter PDFs at three temperatures. Due to an increased $N_{\mathrm{d}}$ and $P_{\mathrm{a}}$, an avalanche is triggered faster at higher temperatures. Consequently, a lower standard deviation of the jitter PDFs can be observed at higher temperatures.

The quantum efficiency for high energy photons emitted from AMLEDs has a negative temperature coefficient [22], therefore a higher $Q_{\mathrm{b}}$ would be required at higher temperatures to ensure the same number of photons at the receiver of an optical link.

\footnotetext{
${ }^{1}$ We focused only on the transmitter, in general $Q_{\mathrm{b}}$ is also lower limited because of the signal-to-noise ratio requirements at the receiver side.
}

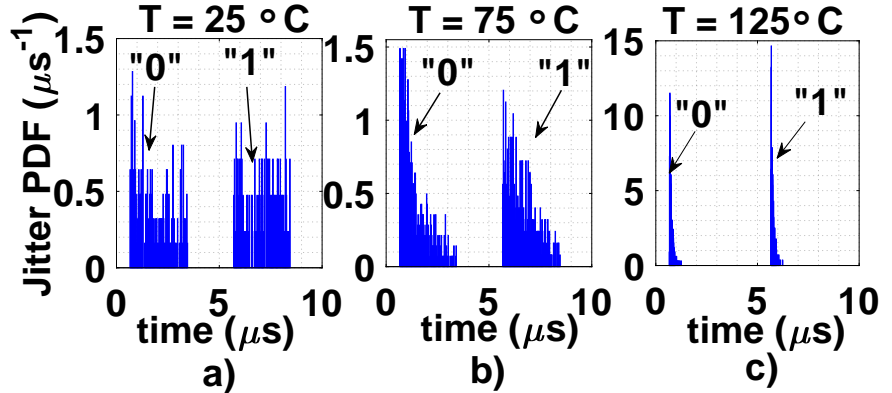

Fig. 13. Measured PDFs of jitter for bit " 0 " and bit " 1 " for $\mathrm{J} 12$ at three temperatures. At all temperatures, $V_{\mathrm{BIAS}}=V_{\mathrm{BR}}+5.9 \mathrm{~V}, V_{\mathrm{L} 1}=V_{\mathrm{BR}}-2.1 \mathrm{~V}$ and $C_{\mathrm{Q}}=470 \mathrm{pF}$. Vertical scales are different for clarity.

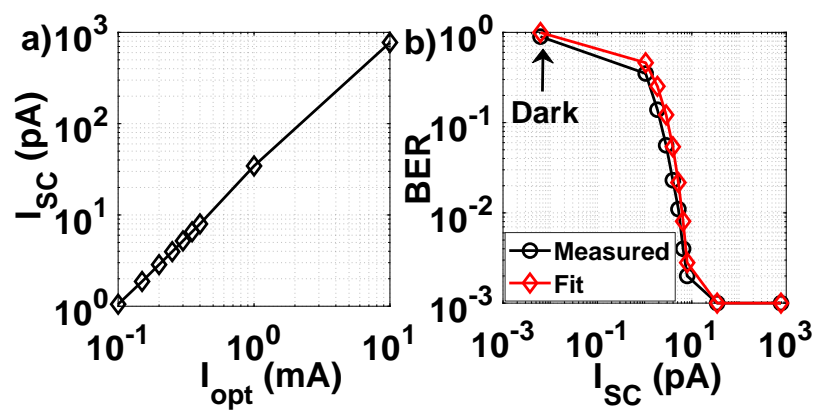

Fig. 14. a) Measured short circuit current $\left(I_{S C}\right)$ in response to current in the external illumination source $\left(I_{\mathrm{opt}}\right)$. (b) Measured and fitted BER as a function of $I_{\mathrm{SC}}$. All axes are on a $\log$ scale.

\section{F. Effect of external illumination}

To demonstrate the effect of increasing $N_{\mathrm{d}}$ without changing $P_{\mathrm{a}}$, free carriers were generated using optical illumination. An increasing $N_{\mathrm{d}}$ results in a lower BER (Eq. 4(d)).

An external LED with an emission spectrum around 650 nm was used to illuminate $\mathrm{J} 12$ at $T=25^{\circ} \mathrm{C}$. Fig. 14(a) shows the measured short circuit current $\left(I_{\mathrm{SC}}\right)$ of $\mathrm{J} 12$ as a function of the external LED current $\left(I_{\mathrm{opt}}\right)$. $I_{\mathrm{SC}}$ is the measured current through the AMLED at $V_{\mathrm{R}}=0$. For higher $I_{\mathrm{opt}}$, more carriers are generated optically and therefore $I_{\mathrm{SC}}$ increases [23]. This implies a higher $N_{\text {opt }}$ and therefore a higher $N_{\mathrm{d}}$ (Eq. 4(b)).

Fig. 14(b) shows the measured BER as a function of $I_{\mathrm{SC}}$ when the driver circuit was operated at $V_{\mathrm{BIAS}}=17.6 \mathrm{~V}, V_{\mathrm{DD}}=$ $6 \mathrm{~V}$ and $C_{\mathrm{Q}}=470 \mathrm{pF}$. For comparison, Eq. (4d) is also plotted with $N_{\mathrm{d}} \approx I_{\mathrm{SC}} T_{\mathrm{ON}} / q_{\mathrm{e}}$ and a fitting parameter $\eta=7 \times 10^{12}$, showing good agreement with the measurements.

The corresponding jitter PDFs in Fig. 15 demonstrate a lower standard deviation for higher $I_{\mathrm{SC}}$, also because of a higher $N_{\mathrm{d}}$. The standard deviation of the jitter was estimated to be $\sim 10$ ns at $I_{\mathrm{SC}}=774.5 \mathrm{pA}$.

\section{G. Impact on light output}

For an optical link, the photon transmission to a nearby photodetector is important. We have already shown a linear relation between $Q_{\mathrm{b}}$ and the emitted photon flux from an AMLED [5], [18]. Therefore, triggering of an avalanche during a data bit impacts the light emission from an AMLED for that bit [18], [24].

A high BER in the transmitted data implies that light would not be emitted from an AMLED during the data bit transfer. 

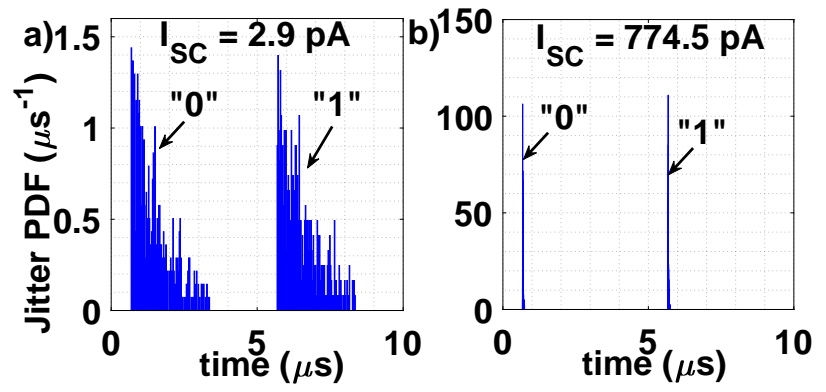

Fig. 15. Example of measured PDF of jitter for "0" and "1" at two $I_{\text {SC }}$ settings. The driver circuit was operated at the same bias settings as in Fig. 14(b). Y-axis scales are different for clarity.

Assuming an ideal receiver, this would result in a high BER of the overall optical link, which is obviously undesired.

Furthermore, a high jitter in the turn-on time of an avalanche would cause a high jitter in the light output from an AMLED. This would limit the application of AMLEDs in optical links using time critical modulation schemes, such as PPM [19].

\section{Design ReCOMmendations}

From the results in section III, we can conclude that jitter and BER in AMLEDs can be reduced through some design techniques. We discuss some of the design recommendations for high speed AMLEDs.

\section{A. Effect of defect density}

By the use of electron beam induced scanning electron microscopy it was shown [25] that avalanche is favored at the defect sites, indicating that defect locations are suitable for the triggering of avalanche. In modern CMOS technologies, the defect density can be increased by increasing the proximity of the depletion region to oxide interfaces, such as shallow trench isolation (STI) [26].

To demonstrate the effect of STI, a $\mathrm{p}^{+} \mathrm{n}$ AMLED with an STI interface and another device without an STI interface were selected from a $140 \mathrm{~nm}$ SOI CMOS technology. Fig. 16(a) shows the schematic cross-sections for these devices, denoted as S7 and C12 [27]. S7 is square in shape with an edge length of $7 \mu \mathrm{m}$, whereas $\mathrm{C} 12$ is a circular device with a diameter of $12 \mu \mathrm{m}$.

Fig. 16(b) shows the $I-V$ characteristics for these AMLEDs. The different $V_{\mathrm{BR}}$ for $\mathrm{S} 7$ and $\mathrm{C} 12$ can be attributed to the different doping of the n-layer in these devices. Assuming one-sided abrupt junctions, the n-well doping in S7 and $\mathrm{C} 12$ is estimated as $6 \times 10^{16}$ and $9 \times 10^{16} \mathrm{~cm}^{-3}$ respectively [23]. Fig. 16(b) shows an example of the light emission from these devices. The PPM speed of these AMLEDs was measured using the same driver circuit and PRBS data (Fig. 6). Figs. 16(c) and 16(d) show examples of the measured transients at the indicated bias settings. As the $V_{\mathrm{EX}, \mathrm{i}}$ at these bias settings is almost same $(\sim 4.9 \mathrm{~V})$, the difference in the peak current of the transients can be explained by the lower resistance of $\mathrm{C} 12$ due to a higher n-well doping, combined with geometric effects [23]. Figs. 16(e) and 16(f) show examples of the measured PDFs of jitter in S7 and C12 respectively.
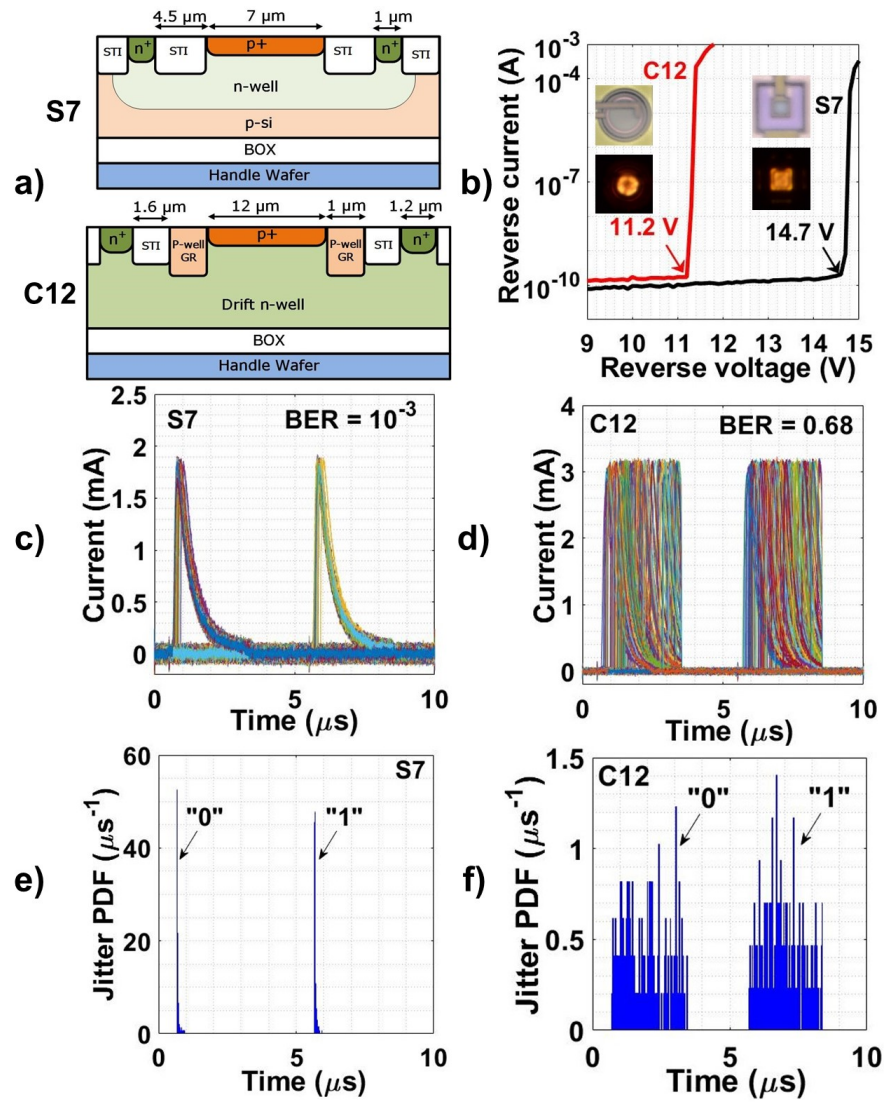

Fig. 16. (a) Schematic cross-section of a square shaped AMLED (S7) and a circular AMLED (C12) in a $140 \mathrm{~nm}$ SOI CMOS technology. (b) Reverse $I-V$ characteristics measured using a Keysight B2901A SMU with $1 \mathrm{~s}$ integration time. The light emission from these AMLEDs, biased at a reverse current of 1 $\mathrm{mA}$, is also shown. The emission was captured using a Nikon D3100 camera with an integration time of $30 \mathrm{~s}$. (c) An example of the measured transients for S7 for $2^{10}$ bits when the driver circuit was operated with $V_{\mathrm{BIAS}}=19.6 \mathrm{~V}$, $V_{\mathrm{DD}}=5 \mathrm{~V}$ and $C_{\mathrm{Q}}=470 \mathrm{pF}$. (d) Measured transients for C12 at $V_{\mathrm{BIAS}}=16.1$ $\mathrm{V}, V_{\mathrm{DD}}=5 \mathrm{~V}$ and $C_{\mathrm{Q}}=470 \mathrm{pF}$. (e,f) Measured PDFs of jitter for bit "0" and bit " 1 " at the same bias settings for the same devices.

For S7, the minimum attainable BER of $10^{-3}$ (section III) and a standard deviation of jitter of $\sim 37 \mathrm{~ns}$ were measured. The measured energy-per-bit at a data rate of $100 \mathrm{kbps}$ was $\sim 23.8 \mathrm{~nJ} /$ bit. In comparison, $\mathrm{C} 12$ showed a higher BER and jitter. These results demonstrate that an STI interface at the junction can help AMLEDs to achieve a higher speed.

\section{B. Other recommendations}

A three terminal AMLED structure was proposed and demonstrated [28] to increase its quantum efficiency. The basic idea is that the third terminal (emitter) injects more carriers, hence higher $N_{\mathrm{d}}$, in the depletion region thereby triggering avalanche, which results in a low BER and jitter. Consequently, such a device concept results in high speed.

Another so-called $\mathrm{n}^{+} \mathrm{pn}^{+} \mathrm{pn}^{+}$device structure has been proposed to increase the efficiency of an AMLED [29]. This structure comprises two reverse biased light-emitting junctions and two forward-biased junctions to increase intra-band transitions by providing extra carriers. Such a device structure could also improve the speed. 
Ideally, for a high speed AMLED, the maximum attainable speed is only limited by the time constants of the driver circuit (and not by triggering speed of avalanche in AMLEDs), which can be in the range of tens of picoseconds in modern CMOS technologies. This could result in a maximum attainable speed in the Gbps range. Furthermore, the ultimate achievable speed is limited by the small-signal modulation speed, which has been shown to be in the range of tens of $\mathrm{GHz}$ [8], [9].

\section{CONCLUSIONS}

The data transmission capabilities of silicon avalanche mode LEDs (AMLEDs) fabricated in CMOS was investigated. The data were modulated using pulse position modulation (PPM) and the bit-error-rate (BER) and the jitter in the transmitted data were measured. The results were correlated to the multiplication noise $\left(\sigma_{\mathrm{M}}\right)$ and the leakage current of the AMLEDs. The $\sigma_{M}$ of these AMLEDs increases with their size because of more defects in larger devices. It was shown that AMLEDs with a higher $\sigma_{M}$ display a lower BER. Through temperature and optical excitations, it was shown that the leakage current also improves the triggering of avalanche, thereby reducing BER and jitter. A PPM speed of $100 \mathrm{kbps}$ at the energy consumption of $23.8 \mathrm{~nJ} / \mathrm{bit}$ has been obtained from the highspeed AMLEDs. The triggering rate can be improved using AMLED designs to inject more carriers in the depletion region and some recommendations for such high speed AMLED designs were proposed. The presented results are important for the design of low power monolithic optical links.

\section{ACKNOWLEDGMENT}

The authors would like to acknowledge Dr. M.-J. Lee and Dr. E. Charbon for the design of device C12, Dr. L. Qi for the pure boron devices, NXP semiconductors for fabricating the SOI devices, H. de Vries for help with the experiments and Dr. J. Schmitz for a critical review of the manuscript.

\section{REFERENCES}

[1] B.P. van Drieënhuizen and R.F. Wolffenbuttel, "Optocoupler based on the avalanche light emission in silicon," Sensors and Actuators A, 31, pp. 229 - 240, 1992, 10.1016/0924-4247(92)80110-O.

[2] L.W. Snyman, H. Aharoni, A. Biber, A. Bogalecki, L. Canning, M. du Plessis and P. Maree, "Optical sources, integrated optical detectors, and optical waveguides in standard silicon CMOS integrated circuitry," Proc. SPIE, vol. 3953, pp. 20-36, 2000, 10.1117/12.379613.

[3] K. Xu, K. A. Ogudo, J.-L. Polleux, C. Viana, Z. Ma, Z. Li, Q. Yu, G. Li and L. W. Snyman, "Light Emitting Devices in Si CMOS and RF Bipolar Integrated Circuits," LEUKOS, vol. 12, no. 4, pp. 203-212, 2016, 10.1080/15502724.2015.1134333.

[4] K. Xu, L. W. Snyman, J.-L. Polleux, K. A. Ogudo and C. Viana, Q. Yu and G. P. Li, "Silicon LEDs toward high frequency on-chip link," Optik, vol. 127, pp. 7002-7020, 2016, 10.1016/j.ijleo.2016.05.025.

[5] S. Dutta, V. Agarwal, R.J.E. Hueting, J. Schmitz and A.J. Annema, "Monolithic optical link in silicon-on-insulator CMOS technology," Opt. Express, vol. 25, no. 5, pp. 5440-5456, 2017, 10.1364/OE.25.005440.

[6] B. Razavi, "Design of integrated circuits for optical communications," p. 58, (McGraw-Hill Inc., 2003).

[7] K. Gingerich and C. Sterzik, "The ISO72x Family of High-Speed Digital Isolators," Application Report SLLA198, Texas Instruments (2006).

[8] A. Chatterjee, B. Bhuva, and R. Schrimpf, "High-speed light Modulation in avalanche breakdown mode for Si diodes," IEEE Electron Device Lett., vol. 25, no. 9, pp. 628 - 630, 2004, 10.1109/LED.2004.834247.

[9] K. Xu, "Integrated Silicon Directly Modulated Light Source Using pWell in Standard CMOS Technology," IEEE Sens. J., vol. 16, no. 16, pp. 6184 - 6191, 2016, 10.1109/JSEN.2016.2582840.
[10] K. Xu, "Electro-Optical Modulation Processes in Si-PMOSFET LEDs Operating in the Avalanche Light Emission Mode," IEEE Tran. Electron Devices, vol. 61, no. 6, pp. 2085 - 2092, 2014, 10.1109/TED.2014.2318277.

[11] B. Huang, X. Zhang, W. Wang, Z. Dong, N. Guan, Z. Zhang, and H. Chen, "CMOS monolithic optoelectronic integrated circuit for on-chip optical interconnection," Opt. Commun., vol. 284, no. 16-17, pp. 39243927, 2011, 10.1016/j.optcom.2011.04.028.

[12] L. Qi, K. R. C. Mok, L. K. Nanver, M. Aminian and E. Charbon, "UV-sensitive low dark-count PureB single-photon avalanche diode," IEEE Trans. Electron Devices, vol. 61, no. 11, pp. 3768-3774, 2014, 10.1109/ICSENS.2013.6688603.

[13] V. Agarwal, A. J. Annema, S. Dutta, R. J. E. Hueting, L. K. Nanver and B. Nauta, "Random Telegraph Signal phenomena in avalanche diodes: Application to SPADs," $46^{\text {th }}$ European Solid-State Device Research Conference (ESSDERC), Lausanne, pp. 264-267, 2016, 10.1109/ESSDERC.2016.7599636.

[14] V. Agarwal, A. J. Annema, S. Dutta, R. J. E. Hueting, L. K. Nanver and B. Nauta, "Random Telegraph Signal phenomena in ultra shallow $\mathrm{p}^{+} \mathrm{n}$ avalanche diodes," IEEE J. Electron Devices Society, vol. 6, no. 1, pp. 642-652, 2018, 10.1109/JEDS.2018.2835153.

[15] R. J. McIntyre, "Multiplication noise in uniform avalanche diodes," IEEE Trans. Electron Devices, vol. ED-13, no. 1, pp. 164-168, 1966, 10.1109/T-ED.1966.15651.

[16] M. J. Kirton and M. J. Uren, "Noise in solid state microstructures: A new perspective on individual defects, interface states and low frequency (1/f) noise," Adv. in Phys., vol. 38, no. 4, pp. 367-468, 1989, $10.1080 / 00018738900101122$.

[17] R. Newman, "Visible light from a silicon p-n junction," Phys. Rev., vol. 100, no. 2, pp. 700-703, 1955, 10.1103/PhysRev.100.700.

[18] V. Agarwal, S. Dutta, A. J. Annema, R. J. E. Hueting, P. G. Steeneken and B. Nauta, "Low power wide spectrum optical transmitter using avalanche mode LEDs in SOI CMOS technology," Opt. Express, vol. 25, no. 15, pp. 16981-16995, 2017, 10.1364/OE.25.016981.

[19] C. Favi and E. Charbon, "Techniques for fully integrated intra-/interchip optical communication," in Proc. 45th ACM/IEEE Design Automation Conf., pp. 343 - 344, 2008, 10.1145/1391469.1391558.

[20] A. Gallivanoni, I. Rech and M. Ghioni, "Progress in Quenching Circuits for Single Photon Avalanche Diodes," IEEE Trans. on nuclear science, vol. 57, no. 6, pp. 3815-3826, 2010, 10.1109/TNS.2010.2074213.

[21] Y. Kang, H. X. Lu, Y.-H. Lo, D. S. Bethune and W. P. Risk, "Dark count probability and quantum efficiency of avalanche photodiodes for singlephoton detection," Appl. Phys. Lett., vol. 83, no. 14, pp. 2955-2957, 2003, 10.1063/1.1616666.

[22] M. du Plessis, H. Wen, and E. Bellotti, "Temperature characteristics of hot electron electroluminescence in silicon," Opt. Exp., vol. 23, no. 10, pp. 12605-12612, 2015, 10.1364/OE.23.012605.

[23] S. M. Sze and K. K. Ng, "Physics of semiconductor devices," Wiley, 2007.

[24] K. Xu, "Monolithically integrated Si gate-controlled light-emitting device: science and properties," Jrnl. Opt., vol. 20, no. 2, pp. 024013, 2018, 10.1088/2040-8986/aaa2b7.

[25] L. W. Snyman, H. Aharoni and M. du Plessis, "Characterization of breakdown phenomena in light emitting silicon $\mathrm{n}^{+} \mathrm{p}$ diodes," J. Appl. Phys., vol. 84, no. 5, pp. 2953-2959, 1998, 10.1063/1.368401.

[26] J. A. Richardson, E. A. G. Webster, L. A. Grant and R. K. Henderson, "Scaleable single-photon avalanche diode structures in nanometer CMOS technology," IEEE Trans. Electr. Dev., vol. 58, no. 7, pp. 20282035, 2011, 10.1117/12.884097.

[27] M. J. Lee, P. Sun, and E. Charbon, "A first single-photon avalanche diode fabricated in standard SOI CMOS technology with a full characterization of the device," Opt. Express, vol. 23, no. 10, pp. 13200-13209, 2015, 10.1364/OE.23.013200.

[28] L. W. Snyman and M. du Plessis and H. Aharoni, "Injection-AvalancheBased $\mathrm{n}^{+}$pn Silicon Complementary Metal Oxide Semiconductor Light Emitting Device $(450$ - $750 \mathrm{~nm})$ with 2-Order-of-Magnitude Increase in Light Emission Intensity," Jap. J. Appl. Phys., vol. 46, no. 4B, pp. 2474-2480, 2007, 10.1143/JJAP.46.2474.

[29] K. Xu, L. Huang, Z. Zhang, J. Zhao, L. W. Snyman and J. W. Swart, "Light emission from a poly-silicon device with carrier injection engineering," Mat. Sci. Eng.: B, vol. 231, pp. 28 - 31, 2018, 10.1016/j.mseb.2018.07.002. 